\title{
Mathematical modeling of thruster vectored AUV based on the single rotating object
}

\author{
Yuehua Huang ${ }^{1, a}$, Peipei Yang ${ }^{2, b}$, Hao Gong ${ }^{3, c}$, Yu Huang ${ }^{4, d}$ \\ ${ }^{1}$ College Of Electrical Engineering \& New Energy, China Three Gorges University, Yichang, Hubei, \\ 443000, China \\ ${ }^{2}$ College Of Electrical Engineering \& New Energy, China Three Gorges University, Yichang, Hubei, \\ 443000, China \\ ${ }^{3}$ College Of Electrical Engineering \& New Energy, China Three Gorges University, Yichang, Hubei, \\ 443000, China \\ ${ }^{4}$ College Of Electrical Engineering \& New Energy, China Three Gorges University, Yichang, Hubei, \\ 443000, China \\ a308001859@qq.com, byangbei263@163.com, “455481603@qq.com, d378752916@qq.com
}

Keywords: thrust vectoring; Autonomous Underwater Vehicle; mathematical modeling

Abstract. The paper makes a kinematics and dynamics analysis of thruster vectored Autonomous Underwater Vehicle based on the structure and principle of vector thruster; and then makes a mathematical model of this thrust vectoring Autonomous Underwater Vehicle based on the classical Momentum and angular momentum theorem. At last a simulation of its motion parameters id made, the result of simulation shows, the parameters response speed in the way of thruster vectored Autonomous Underwater Vehicle is faster than that in the way of ordinary vectored Autonomous Underwater Vehicle.

\section{Introduction}

Autonomous Underwater Vehicle (AUV) is a kind of robot that has characteristics of intelligence and independent navigation. It was called as naval force multiplier and has a broad military purpose. Typically, an AUV with general thruster is controlled by rudders and fins and the desired force is supplied by operating rudder, but its efficiency can be reduced. To solve this problem, domestic and foreign scholars proposed a kind of AUV with vector thruster which can provide required pitching force, pitching moment and yawing moment by changing the direction of propulsion. For example, the Bluefin-12 AUV of American Bluefin Robotics Corporation, the Thales' AUV of British BAE Systems Corporation and Gavia Scientific AUV of Iceland Hafmynd Corporation[1]。This paper proposed a kind of vector thruster AUV, established its mathematical model and carried on simulation with kinematic parameter, and compared with common thruster. The model can be use to simulate the normal cruise state and analyze the maneuverability of the vehicle and design and simulate the control system.

\section{Principle of Vector Thruster}

The vector thruster is composed of the first action mechanism, the second action mechanism, main motor, rib, jointing element, duct and airscrew (shown in Figure 1). Duct is connected with airscrew hub through rib. A whole component namely conduit component is formed by airscrew hub connect duct, airscrew and rib through connector. Conduit component is connected with jointing element through plug(shown in Figure 1 axis 2). The first and second action mechanism can do telescoping movement. The first mechanism can be rotatably connected on connector; when the first action mechanism do telescoping movement, conduit component moves around axis 2 and then the yaw movement of vehicle is formed. The second action mechanism is connected with conduit component through jointing element; when the second action mechanism do telescoping movement, conduit component and jointing element move around axis 2 together and then the pitch movement of vehicle is formed. The vector control of vehicle is achieved by this structure of vector thruster; it can drive motor, 
duct and airscrew to rotate by the two action mechanism do telescoping movement. a little drive torque and high control precision is required when changing the attitude of vehicle[2].

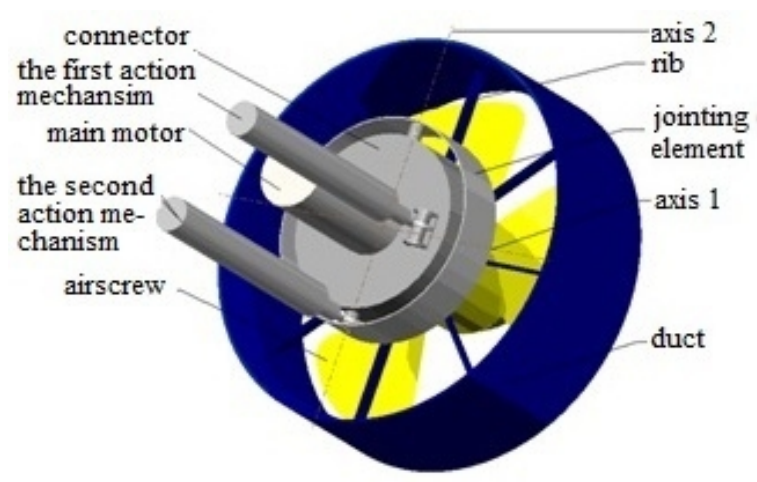

Fig. 1. The diagrammatic drawing of vector thruster of AUV

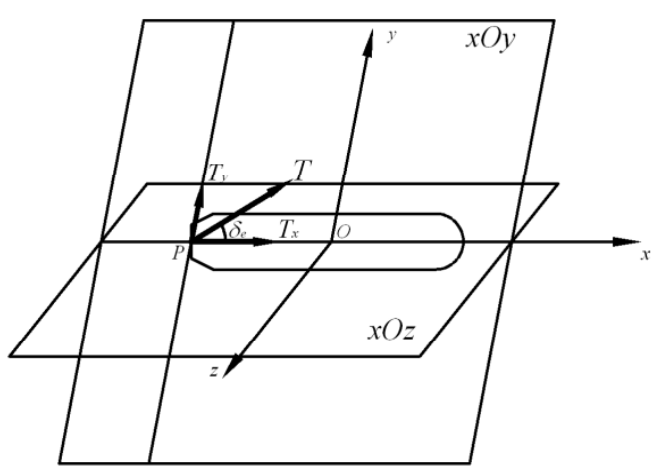

Fig. 2. The breakdonwn drawing of $\mathrm{T}$ when doing pitching motion

\section{The mathematical modeling}

The main distinction of AUV equipped with vector thruster and traditional AUV is the mode of changing vehicle's attitude and whether there is rudder. The vector propulsion vehicle provides the required pitching force, pitching moment and yawing moment by changing the direction of propulsion instead of traditional steering mode offers the required operating force. Thus, we can take the past method of establishing model as a reference during the new modeling, the difference is convert the force and moment which is from the interaction of rudder and water flow to the force and moment provided by the component force of thruster; at the same time, as the tail of AUV equipped vector thruster can shift, the shell of vehicle may be affected by additional resistance when the axis direction of airscrew disk is at odds with the direction of vehicle's velocity.

\section{Coordinate system}

The coordinate system consist of geographic coordinate system $O_{0} x_{0} y_{0} z_{0}$, carrier coordinate system $O x y z$, translate coordinate system $O x^{\prime} y^{\prime} z^{\prime}$ and velocity coordinate system $O x_{1} y_{1} z_{1}$. We take the launching point as original point $O_{0}$ in geographic coordinate system; The $O_{0} x_{0}$ axis points to the east, the $O_{0} y_{0}$ axis points to the north, and the $O_{0} z_{0}$ axis points to the heavens; the three axes consist of the right hand rectangular coordinate system. Analogously, we regard the core of vehicle's buoyancy as the original point $O$ in carrier coordinate system; the $O x$ axis point to the head of vehicle, the $O y$ axis points to above the vehicle's back that is perpendicular to the $O y$ axis, axis $O z$ is perpendicular to the plane composed by $O x$ axis and $O y$ axis; the three also consist of the right hand rectangular coordinate system. The three axes of carrier coordinate system are closed to the centre principal inertia axis of vehicle. The translate coordinate system moves along with vehicle, its origin of coordinates always overlaps with the origin of carrier coordinates, each axis $O x^{\prime}, O y^{\prime}, O z^{\prime}$ are respectively parallel to the axis $O_{0} x_{0}, O_{0} y_{0}, O_{0} z_{0}$ of geographic coordinate system during the movement of vehicle and their directions are the same. The origin of velocity coordinates also overlaps with the origin of carrier coordinates; the $O x_{1}$ axis overlaps with velocity vector of the origin of vehicle, the $O y_{1}$ axis is located in the longitudinal plane of symmetry of vehicle and perpendicular to $O x_{1}$ axis, and pointing up; $O z_{1}$ axis is perpendicular to the plane of $x_{1} O y_{1}$; the three axes follow the rule of right hand rectangular coordinate system. 


\section{Kinematic parameter}

(1) The position coordinates of vehicle in geographic coordinate system are $x_{0}, y_{0}, z_{0}$.

(2) The velocity vector of the centre of buoyancy of the AUV is $v$, and the angular velocity vector is $w ; v_{x}, v_{y}, v_{z}$ are the components of $v$ in the carrier coordinate system and $v_{x 0}, v_{y 0}, v_{z 0}$ is the component of $v$ in the geographic coordinate system; $w_{x}, w_{y}, w_{z}$ are the components of $w$ in the carrier coordinate system. Anytime, the relationship between $x_{0}, y_{0}, z_{0}$ and the velocity of vehicle in carrier coordinate system is as follows

$$
\frac{d x_{0}}{d t}=v_{x 0}, \frac{d y_{0}}{d t}=v_{y 0}, \frac{d z_{0}}{d t}=v_{z 0}
$$

(3) Attitude angle is $\theta, \psi, \varphi, \theta$ is the pitching angle of vehicle, $\psi$ is the yawing angle and $\varphi$ is the rolling angle.

(4) Attack angle $\partial$ and sideslip angle $\beta$. The relationship between $\partial, \beta$ and the component of velocity of vehicle in carrier coordinate system is as follow

$$
\left\{\begin{array}{l}
\alpha=-\arctan \left(v_{y} / v_{x}\right) \\
\beta=\arctan \left(v_{z} / \sqrt{v_{x}^{2}+v_{y}^{2}}\right)
\end{array}\right.
$$

(5) Declination angle of duct are $\sigma_{e}, \sigma_{r}$. Duct can rotate around vertical axis and horizontal axis in the servo mechanism; namely, duct can has a deflexion in the plane of $x O y$ and $x O z$ of carrier coordinate system. Where, $\sigma_{e}$ is the included angle between the axis of airscrew and the $O x$ axis both on the plane of $x O y$ where duct is in; and $\sigma_{r}$ is the included angle between the axis of airscrew and the $O x$ axis both on the plane of $x O z$ where duct is in.

\section{The movement degree of freedom}

The longitudinal motion of AUV includes forward, ascending, diving and pitching; the lateral movement of AUV covers lateral swaying, yawing and rolling [3].

\section{The kinematical equation of AUV}

According to the transfer matrix that from carrier coordinate system to geographic coordinate system, we can get the parallel movement equation of motion of the centre of buoyancy, shown as follows

$$
\left[\begin{array}{lll}
\dot{x}_{0} & \dot{y}_{0} & \dot{z}_{0}
\end{array}\right]^{T}=C_{0}^{b}\left[\begin{array}{lll}
u_{x} & u_{y} & u_{z}
\end{array}\right]^{T}
$$

Where $C_{0}^{b}$ is the transfer matrix.

The kinematical equation can be obtained on the basis of the transition relation which from geographic coordinate system to carrier coordinate system

$$
\left[\begin{array}{l}
w_{x} \\
w_{y} \\
w_{z}
\end{array}\right]=\left[\begin{array}{ccc}
\sin \theta & 0 & 1 \\
\cos \theta \cos \varphi & \sin \varphi & 0 \\
-\cos \theta \sin \varphi & \cos \varphi & 0
\end{array}\right]\left[\begin{array}{c}
\dot{\psi} \\
\dot{\theta} \\
\dot{\varphi}
\end{array}\right]
$$

\section{The dynamical equation of AUV}

The coordinate of the core of AUV in carrier coordinate system which origin is the centre of buoyancy is $r_{G}=\left[\begin{array}{lll}x_{G} & y_{G} & z_{G}\end{array}\right]^{T}$. Assuming the mass of AUV is not changing with time, and the rotate of earth is ignored; we suppose the shape of AUV is centered on both the vertical plane of $x O y$ and transversal 
plane of $x \mathrm{Oz}$. On the basis of the theorem of both momentum and momentum moment, the dynamical equation of six degree of freedom of rigid body in carrier coordinate system is as follows [4]

$$
\left\{\begin{array}{l}
\frac{d Q}{d t}+w \times Q=F \\
\frac{d K}{d t}+w \times K+v_{0} \times Q=M
\end{array}\right.
$$

Where $F$ and $M$ are the projection of external force and its moment on carrier coordinate system.

\section{The force and its moment on AUV}

(1) Gravity $G$ and buoyancy $B$

(2)Thrust $T$

For a general underwater vehicle, only one main airscrew thruster which generates thrust $T$ along with the axis $O x$ of carrier coordinate system is installed on the tail of vehicle along the direction of vertical axis [5].

But for vector thruster whose thrust is a vector, its direction of thrust can be appropriately adjusted according to need of control of vehicle, instead of simplex along with the direction of $O x$ axis of carrier coordinate system; additionally, due to airscrew can only sway in the plane of $x O y$ and $x O z$ of carrier coordinate system under the thrust of piston.

Thrust $T$ could be decomposed into $T=\left[\begin{array}{lll}T_{x} & T_{y} & 0\end{array}\right]$ (shown as figure 2) when AUV do pitching motion; where $T_{x}, T_{y}$ are the components of $T$ along the direction of $O x, O y$, respectively. $\sigma_{e}$ is the included angle between the axis of airscrew and the $O x$ axis both on the plane of $x O y$ where duct is in. Assuming the angle is positive when airscrew is downward-skewed, and then there will be $T_{x}=T \cos \delta_{e}, T_{y}=T \sin \delta_{e}, T_{z}=0$. The pitching oment $M=-\left|T_{y}\right||\overline{P O}|$, where $\overline{P O}$ is the distance of action spot to the buoyancy centre.

Thrust $T$ could be decomposed into $T=\left[\begin{array}{lll}T_{x} & 0 & T_{z}\end{array}\right]$ (shown as figure 3) when AUV do yawing motion; where, $T_{x}, T_{z}$ are the components of $T$ along the direction of $O x 、 O z$, respectively. ${ }^{\sigma_{r}}$ is the included angle between the axis of airscrew and the $O x$ axis both on the plane of $x O z$ where duct is in. Assuming the angle is positive when airscrew is right-skewed, and then there will be $T_{x}=T \cos \delta_{r}, T_{y}=0, T_{z}=-T \sin \delta_{r}$. yawing moment $N=-\left|T_{z}\right||\overline{P O}|$.

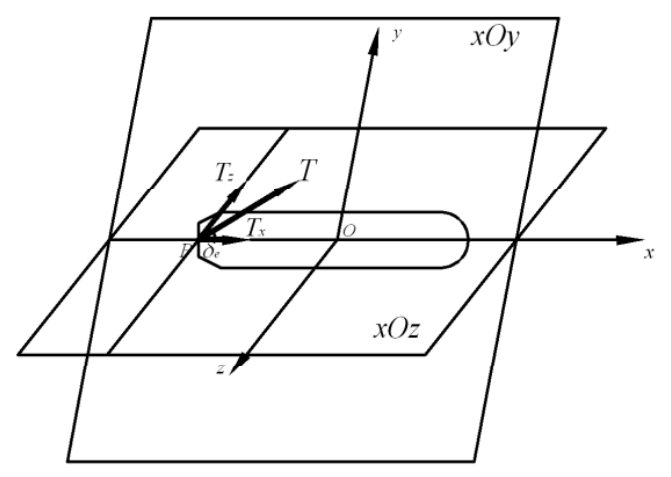

Fig. 3. The breakdonwn drawing of $\mathrm{T}$ when doing yawing motion.

(3) Dynaflow

For definitive fluid medium and defined outline AUV, the dynaflow and moment only depend on the motion parameters which can be getting from experiment. The specific calculation method of dyanflow and its moment, including position force, damping force and inertia force, can refer to reference[6]. 


\section{AUV Space Motion Equation}

(1) Longitudinal motion equation.

$\beta=v_{z}=w_{x}=w_{y}=\psi=\varphi=z_{0}=0$, on the basis of motion equation(3) and kinetic equation (4) and the force analysis on AUV, the reduced equation of longitudinal motion can be shown as equation (6). (2) Lateral motion equation.

$v_{y}=w_{z}=y_{0}=0$, the value of $\theta, \alpha$ is small. Assuming that $\sin \theta=\sin \alpha=0, \cos \theta=\cos \alpha=1$, the reduced equation of lateral motion can be shown as equation (7).

$$
\begin{aligned}
& \left(\left(m+\lambda_{11}\right) \dot{v}_{x}=T \cos \delta_{e}-\frac{1}{2} C_{x} \rho v^{2} S-\Delta G \sin \theta\right. \\
& \left(m+\lambda_{22}\right) \dot{v}_{y}+\left(m x_{G}+\lambda_{26}\right) \dot{w}_{z}=-m v_{x} w_{z}+\frac{1}{2} \rho v^{2} S c_{y}^{\alpha} \alpha+T \sin \delta_{e}+\frac{1}{2} \rho v^{2} S c_{y_{1}}^{w} w_{z} L / v-\Delta G \cos \theta \\
& \left\{\begin{array}{l}
\left(J_{z z}+\lambda_{66}\right) \dot{w}_{z}+\left(m x_{G}+\lambda_{26}\right) \dot{v}_{y}=-m x_{G} v_{x} w_{z}+G\left(y_{G} \sin \theta-x_{G} \cos \theta\right)+\frac{1}{2} \rho v^{2} S L\left(m_{z_{1}}^{\alpha} \alpha+m_{z_{1}}^{\alpha} w_{z}\right)-T \sin \delta_{e} \overline{P O} \\
\dot{\theta}=w_{z}
\end{array}\right. \\
& \dot{\theta}=w_{z} \\
& \dot{x}_{0}=v_{x} \cos \theta-v_{y} \sin \theta \\
& \dot{y}_{0}=v_{x} \sin \theta+v_{y} \cos \theta \\
& \alpha=-\arctan \left(v_{y} / v_{x}\right) \\
& v=\sqrt{v_{x}^{2}+v_{y}^{2}} \\
& \left(m+\lambda_{11}\right) \dot{v}_{x}=T \cos \delta_{r}-\frac{1}{2} C_{x} \rho v^{2} S \\
& \left(m+\lambda_{33}\right) \dot{v}_{z}-\left(m x_{G}-\lambda_{35}\right) \dot{w}_{y}=m v_{x} w_{y}+\frac{1}{2} \rho v^{2} S c_{z}^{\beta} \beta-T \sin \delta_{r}+\frac{1}{2} \rho v^{2} S c_{z_{1}}^{w} w_{y} L / v \\
& \left\{\begin{array}{l}
\left(J_{y y}+\lambda_{55}\right) \dot{w}_{y}-\left(m x_{G}-\lambda_{35}\right) \dot{v}_{z}=-m x_{G} v_{x} w_{y}+\frac{1}{2} \rho v^{2} S L\left(m_{y_{1}}^{\beta} \beta+m_{y_{1}}^{w} w_{y} L / v\right)-T \sin \delta_{r} \overline{P O} \\
\dot{\psi}=w_{y}
\end{array}\right. \\
& \dot{x}_{0}=v_{x} \cos \psi+v_{z} \sin \psi \\
& \dot{z}_{0}=-v_{x} \sin \psi+v_{z} \cos \psi \\
& \beta=\arctan \left(v_{z} / v_{x}\right) \\
& v=\sqrt{v_{x}^{2}+v_{z}^{2}}
\end{aligned}
$$

Where, $C_{x}, c_{z}^{\beta}, c_{y}^{\alpha}, c_{z_{1}}^{w}, c_{y_{1}}^{w}, m_{y_{1}}^{\beta}, m_{y_{1}}^{w}$ are the dynaflow parameters; $\lambda_{11}, \lambda_{22}, \lambda_{26}, \lambda_{33}, \lambda_{35}, \lambda_{44}, \lambda_{55}, \lambda_{66}$ are additional mass.

\section{Simulation analyses}

A certain boundary dimension of vehicle with 533 caliber and the parameters of dynaflow are adopted to simulate, the specific value and meaning can be seen reference [7].

Supposing that the system motion without disturbance is stationary motion, the speed in balance angle of attack is $v=v_{x}=3 \mathrm{~m} / \mathrm{s}$, the distance from the action of thrust to the centre of buoyancy is $\overline{P O}=3 \mathrm{~m}$; the motion of AUV can be decomposed to longitudinal motion and transverse motion, and the influence of rolling is ignored. The lateral displacement when doing longitudinal motion is $z_{0}=0$, the angular velocity is $w_{x}=w_{y}=0$, yawing angle and rolling angle are $\psi=\varphi=0$, sideslip angle is $\beta=0$. The longitudinal displacement when doing lateral motion is $y_{0}=0$, the angular velocity 
is $w_{z}=0$, pitching angle is $\theta=0$, attacking angle is $\alpha=0$. According to the longitudinal motion equation(6) and the lateral motion equation (7), we can get the transfer function both of longitudinal motion parameters and lateral motion parameters. The transition curve of parameters of both longitudinal motion and lateral motion by simulation with Matlab(shown as figure 4 and figure 5).
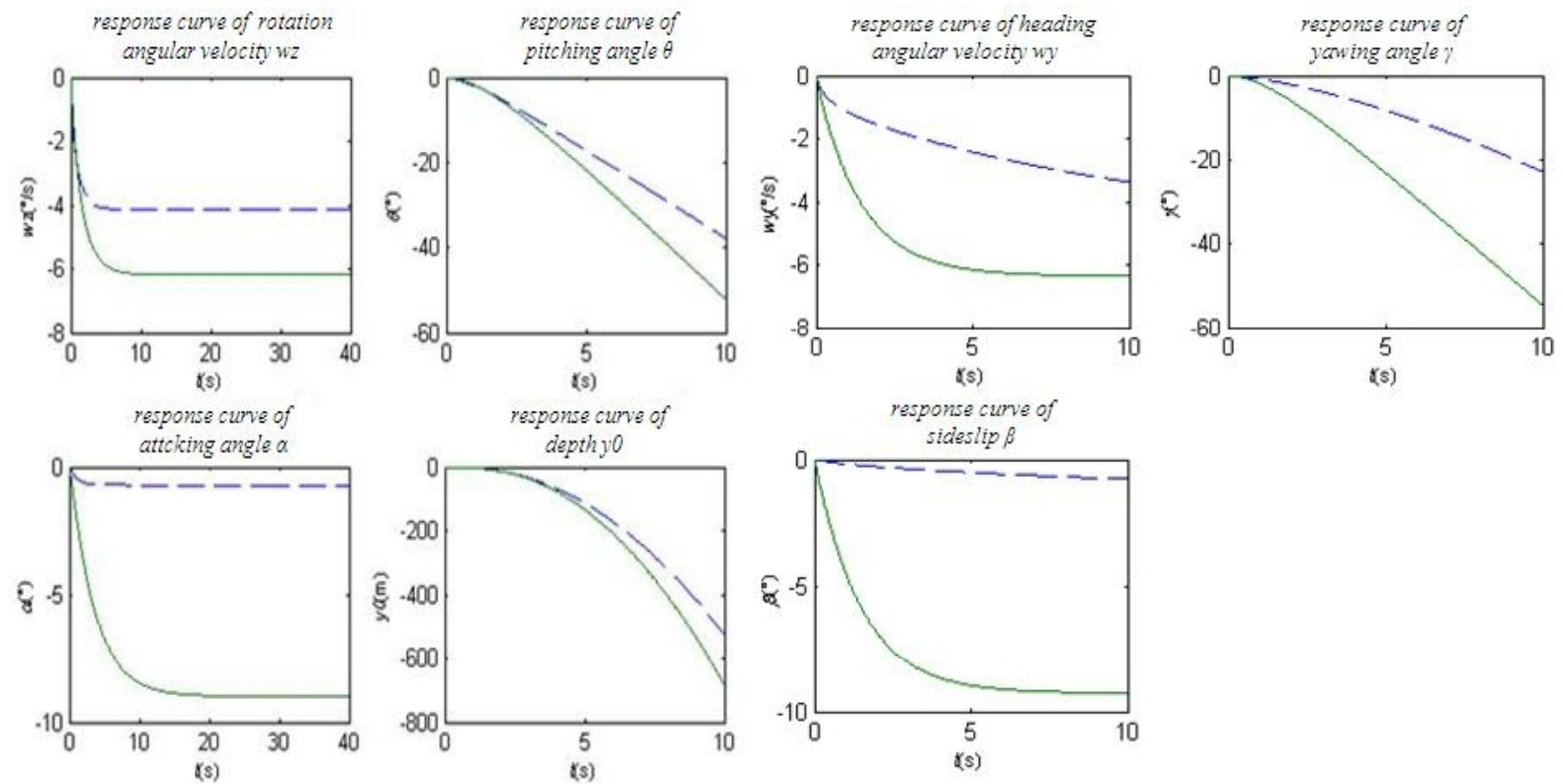

Fig. 4. Transition curves of longitudinal parameters

Fig. 5. Transition curves lateral parameters (Solid wire--the vector propulsion mode; dotted line--common propulsion mode)

As shown above, during the time $t \rightarrow \infty$, the trends of response curves of motion parameters of AUV with no matter the vector propulsion AUV or common propulsion are approximately the same.

When doing longitudinal motion, the rotation angular velocity and attacking angle are converged over time, but pitching angle and depth are diverging over time. When doing lateral motion, heading angular velocity and sideslip angle are converged over time, but heading angle is diverging over time. Additionally, the response rate both of longitudinal motion parameters and lateral motion parameters in the mode of vector propulsion are faster in the mode of common propulsion. We can see the mathematical model conforms to the practical law of motion of AUV with vector propulsion from above analyses.

\section{Conclusions}

This paper introduces a kind of AUV with vector propulsion and makes its mathematical model, then makes simulation for the model. The result of simulation shows, the model we made conforms to the practical law of motion of AUV with vector propulsion, and can be used for both subsequent analysis for operating performance and simulation for controller performance. Control method will be added next, which maintains certain attitude of AUV and satisfies the practical motion demand.

\section{References}

[1] ScottWillcox. Bluefin-12 UUVs for SMCM/UUV-2[R]. Bluefin Robotics Corp.and QinQLtd,2010

[2] Yi Huang. Modeling and simulation for underwater vehicle with vector thruster[C].Northwestern Polytechnical University,2006 "In Chinese" 
[3] Pin Wang. Research on Vector Thruster for Autonomous Underwater Vehicle[D]. Northwestern Polytechnical University, 2006 "In Chinese"

[4] Timothy Prestero. Verification of a six-Degree of Freedom Simulation Model for the REMUS Autonomous Underwater Vehicle [D].Massachusetts Institute of Technology, 2001

[5] WilliamJ. Kirkwood.TAIL ASSEMBLY FOR AN UNDERWATER VEHICLE [J].Patent Application Publication, 2001.6

[6] Weisheng Yan. The torpedo navigation mechanism[M]. Northwestern Polytechnical University,2004 "In Chinese"

[7] Deming Xu. Torpedo Controller System [M]. Northwestern Polytechnical University, 1990 “In Chinese"

[8] Guangyuan Zhao. MATLAB and simulation practice of control system[M].Beijing University of Aeronautics and Astronautics,2012 "In Chinese" 